\title{
Chaotic Motions in the Real Fuzzy Electronic Circuits
}

\author{
Shih-Yu Li, ${ }^{1,2}$ Cheng-Hsiung Yang, ${ }^{3}$ Chin-Teng Lin, ${ }^{2,4}$ Li-Wei Ko, ${ }^{1,2}$ and Tien-Ting Chiu ${ }^{5}$ \\ ${ }^{1}$ Department of Biological Science and Technology, National Chiao Tung University, 1001 Ta Hsueh Road, Hsinchu 300, Taiwan \\ ${ }^{2}$ Brain Research Center, National Chiao Tung University, Hsinchu, Taiwan \\ ${ }^{3}$ Department of Automatic Control, National Taiwan University of Science and Technology, Taipei City, Taiwan \\ ${ }^{4}$ Institute of Electrical Control Engineering, National Chiao Tung University, Hsinchu, Taiwan \\ ${ }^{5}$ Department of Industrial and Systems Engineering, Chung Yuan Christian University, Chung-Li, Taiwan
}

Correspondence should be addressed to Shih-Yu Li; agenghost@gmail.com

Received 26 October 2012; Accepted 30 December 2012

Academic Editor: Chuandong Li

Copyright (C) 2013 Shih-Yu Li et al. This is an open access article distributed under the Creative Commons Attribution License, which permits unrestricted use, distribution, and reproduction in any medium, provided the original work is properly cited.

Fuzzy electronic circuit (FEC) is firstly introduced, which is implementing Takagi-Sugeno (T-S) fuzzy chaotic systems on electronic circuit. In the research field of secure communications, the original source should be blended with other complex signals. Chaotic signals are one of the good sources to be applied to encrypt high confidential signals, because of its high complexity, sensitiveness of initial conditions, and unpredictability. Consequently, generating chaotic signals on electronic circuit to produce real electrical signals applied to secure communications is an exceedingly important issue. However, nonlinear systems are always composed of many complex equations and are hard to realize on electronic circuits. Takagi-Sugeno (T-S) fuzzy model is a powerful tool, which is described by fuzzy IF-THEN rules to express the local dynamics of each fuzzy rule by a linear system model. Accordingly, in this paper, we produce the chaotic signals via electronic circuits through T-S fuzzy model and the numerical simulation results provided by MATLAB are also proposed for comparison. T-S fuzzy chaotic Lorenz and Chen-Lee systems are used for examples and are given to demonstrate the effectiveness of the proposed electronic circuit.

\section{Introduction}

Nonlinear dynamics, commonly called the chaos theory, changes the scientific way of looking at the dynamics of natural and social systems, which has been intensively studied over the past several decades [1-10]. The phenomenon of chaos has attracted widespread attention amongst mathematicians, physicists, and engineers and has also been extensively studied in many fields, such as chemical reactions $[11,12]$, biological systems $[13,14]$, information processing $[15,16]$, and secure communications [17-20].

The mathematical meteorologist Lorenz discovered chaos in a simple system of three autonomous ordinary differential equations in order to describe the simplified Rayleigh-Bénard problem [21] in 1963 which is the most popular system for studying [22-26]. Chen and Lee reported a new chaotic system [27] in 2004, which is now called the Chen-Lee system [28]. The chaotic Chen-Lee system was developed based on the Euler equations for the motion of rigid body. It was proved that this system is the governing set of equations for gyromotion with feedback control. Recently, studies were conducted on this system to explore its dynamic behavior, including the fractional order behavior, the generation of hyperchaos and perturbation analysis, the control and anti-control of chaos, and the synchronization $[29,30]$.

Since the fuzzy set theory [31] and the fuzzy logic [32] were initiated by Zadeh in 1965 and 1973, fuzzy logic has received much attention as a powerful tool for the nonlinear filed. Among various kinds of fuzzy methods, Takagi-Sugeno fuzzy system is widely accepted as a tool for design and analysis of fuzzy control system [33]. The T-S fuzzy model proposes a successful method to deal with certain complex nonlinear systems via some local linear subsystems. There are plenty of researches using the Takagi-Sugeno (T-S) fuzzy model to represent typical chaotic models and then apply some effective fuzzy techniques [34-42]. However, there are still no real 
experimental models in electronic circuit for Takagi-Sugeno (T-S) fuzzy-based chaotic systems. In this paper, we carry out the powerful tool, Takagi-Sugeno (T-S) fuzzy model, in electronic circuit and show good agreement between computer simulations in MATLAB and experimental results in our circuits.

The layout of the rest of the paper is as follows. In Section 2, the Takagi-Sugeno fuzzy model is introduced. In Section 3, experimental results and configurations in electronic circuits for T-S fuzzy chaotic Lorenz and Chen-Lee systems are presented. In Section 4, conclusions are given.

\section{Takagi-Sugeno Fuzzy Model}

In system analysis and design, it is important to select an appropriate model representing a real system. As an expression model of a real plant, we use the fuzzy implications and the fuzzy reasoning method suggested by Takagi and Sugeno. The Takagi-Sugeno (T-S) fuzzy model is described by fuzzy IF-THEN rules which represent local linear input-output relations of a nonlinear system. The main feature of the $\mathrm{T}$ $S$ fuzzy model is to express the local dynamics of each fuzzy rule by a linear system model.

The overall fuzzy model of the system is achieved by fuzzy blending of the linear system models. Consider a continuoustime nonlinear dynamic system as follows.

Rule i:

$$
\begin{aligned}
& \text { IF } x_{1}(t) \text { is } M_{i 1} \cdots \text { and } x_{n}(t) \text { is } M_{i n} \\
& \text { THEN } \dot{x}(t)=A_{i} x(t)+B_{i} u(t)
\end{aligned}
$$

where

$$
\begin{aligned}
& x(t)=\left[x_{1}(t), x_{2}(t), \ldots, x_{n}(t)\right]^{T}, \\
& u(t)=\left[u_{1}(t), u_{2}(t), \ldots, u_{n}(t)\right]^{T},
\end{aligned}
$$

$i=1,2, \ldots, r$ ( $r$ is the number of IF-THEN rules), $M_{i j}$ are fuzzy sets, and $x(t)=A_{i} x(t)+B_{i} u(t)$ is the output from the $i$ th IF-THEN rule. Given a pair of $(x(t), u(t))$, the final output of the fuzzy system is inferred as follows:

$$
\dot{x}=\frac{\sum_{i=1}^{r} \omega_{i}(x(t))\left\{A_{i} x(t)+B_{i} u(t)\right\}}{\sum_{i=1}^{r} \omega_{i}(x(t))},
$$

where

$$
\omega_{i}(x(t))=\prod_{j=1}^{n} M_{i j}(x(t)),
$$

for all $t$, and $M_{i j}(x(t))$ is the grade of membership $x_{j}(t)$ of in $M_{i j}$.

The open-loop system of (3) is

$$
\dot{x}=\frac{\sum_{i=1}^{r} \omega_{i}(x(t)) A_{i} x(t)}{\sum_{i=1}^{r} \omega_{i}(x(t))},
$$

where it is assumed that

$$
\sum_{i=1}^{r} \omega_{i}(x(t))>0, \quad \omega_{i}(x(t)) \geq 0, i=1,2, \ldots, r .
$$

By introducing $h_{i}(x(t))=\omega_{i}(x(t)) / \sum_{i=1}^{r} \omega_{i}(x(t))$ instead of $\omega_{i}(x(t)),(3)$ and (5) can be rewritten as

$$
\begin{aligned}
& \dot{x}=\sum_{i=1}^{r} h_{i}(x(t))\left\{A_{i} x(t)+B_{i} u(t)\right\}, \\
& \dot{x}=\sum_{i=1}^{r} h_{i}(x(t)) A_{i} x(t) .
\end{aligned}
$$

Note that

$$
\sum_{i=1}^{r} h_{i}(x(t))=1, \quad h_{i}(x(t)) \geq 0, i=1,2, \ldots, r,
$$

for all $t . h_{i}(x(t))$ can be regarded as the normalized weight of the IF-THEN rules.

\section{Implementation of T-S Fuzzy Systems on Electronic Circuit}

This section shows the electronic circuit implementations of the T-S fuzzy model of classical Lorenz system and Chen-Lee system. The experimental results are going to be compared with the simulation results given by MATLAB.

3.1. Fuzzy Modeling of Lorenz System. For Lorenz system [21],

$$
\begin{aligned}
& \dot{x}_{1}=a\left(x_{2}-x_{1}\right), \\
& \dot{x}_{2}=c x_{1}-x_{1} x_{3}-x_{2}, \\
& \dot{x}_{3}=x_{1} x_{2}-b x_{3},
\end{aligned}
$$

where $a, b, c$ are the parameters. When $a=10, b=8 / 3, c=$ 28 , and initial states are $(-0.1,0.2,0.3)$, the dynamic behavior is chaotic. Assume that $x_{1} \in[-d, d]$ and $d>0$, then Lorenz system can be exactly represented by T-S fuzzy model as follows [43].

Rule 1:

$$
\begin{aligned}
& \text { IF } x \text { is } M_{1} \text {, } \\
& \text { THEN } \dot{X}(t)=A_{1} X(t) \text {. }
\end{aligned}
$$

Rule 2:

$$
\begin{aligned}
& \text { IF } x \text { is } M_{2}, \\
& \text { THEN } \dot{X}(t)=A_{2} X(t),
\end{aligned}
$$

where

$$
\begin{aligned}
& X=\left[x_{1}, x_{2}, x_{3}\right]^{T}, \\
& A_{1}=\left[\begin{array}{ccc}
-a & a & 0 \\
c & -1 & -d \\
0 & d & -b
\end{array}\right], \quad A_{2}=\left[\begin{array}{ccc}
-a & a & 0 \\
c & -1 & d \\
0 & -d & -b
\end{array}\right] \text {, } \\
& M_{1}(x)=\frac{1}{2}\left(1+\frac{x_{1}}{d}\right), \quad M_{2}(x)=\frac{1}{2}\left(1-\frac{x_{1}}{d}\right) \text {. }
\end{aligned}
$$




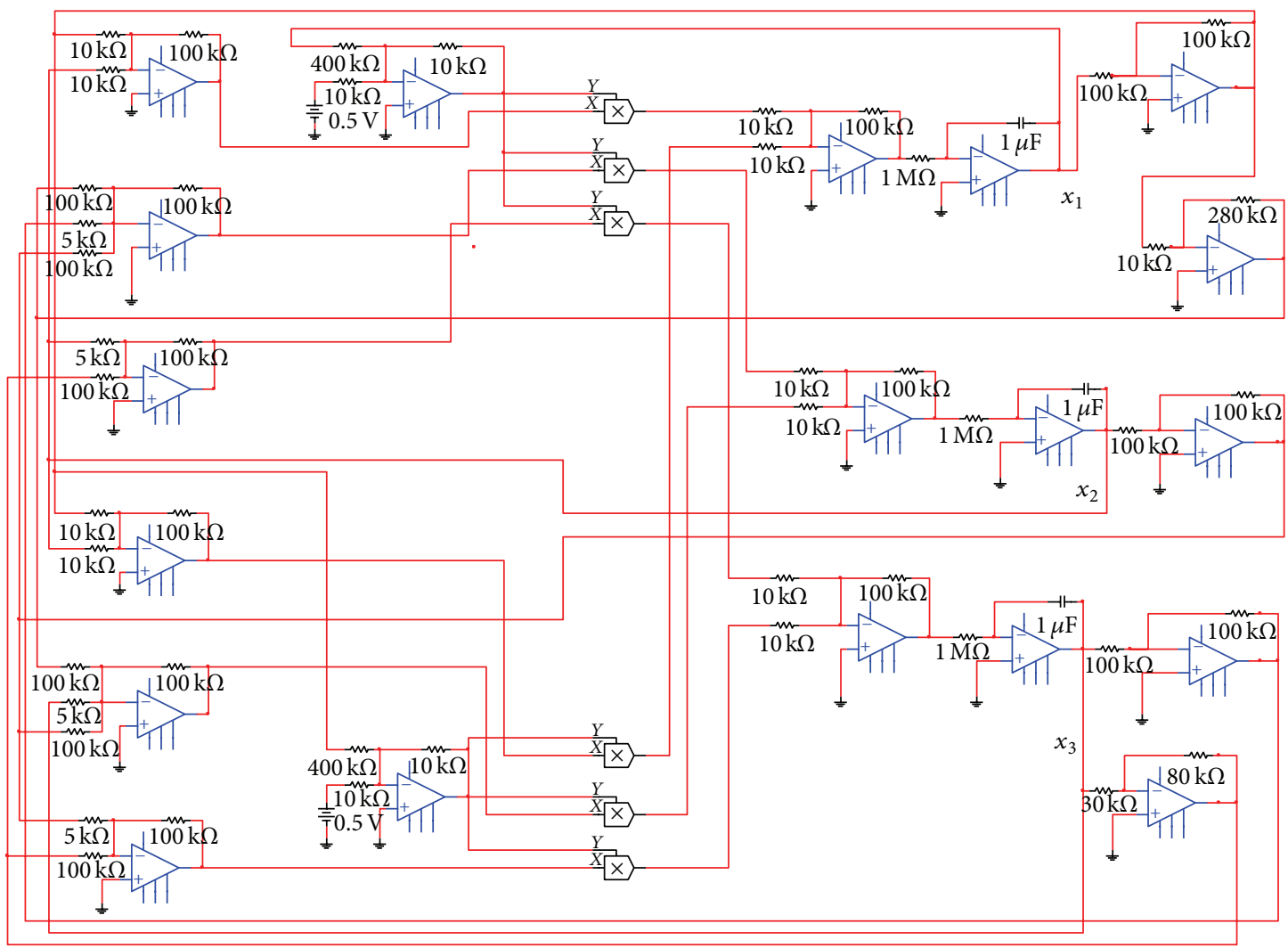

Figure 1: The fuzzy electronic circuit for chaotic Lorenz system.

Choosing $d=30, M_{1}$ and $M_{2}$ are fuzzy sets of Lorenz system. Here, we call (10) the first linear subsystem under the fuzzy rule and (11) the second linear subsystem under the fuzzy rule. The final output of the fuzzy Lorenz system is inferred as follows:

$$
\begin{aligned}
\dot{X}(t) & =\sum_{i=1}^{2} h_{i} A_{i} X(t) \\
& =\left[\begin{array}{l}
h_{1} \\
h_{1} \\
h_{1}
\end{array}\right]^{T}\left[\begin{array}{c}
a\left(x_{2}-x_{1}\right) \\
c x_{1}-d x_{3}-x_{2} \\
d x_{2}-b x_{3}
\end{array}\right]+\left[\begin{array}{l}
h_{2} \\
h_{2} \\
h_{2}
\end{array}\right]^{T}\left[\begin{array}{c}
a\left(x_{2}-x_{1}\right) \\
c x_{1}+d x_{3}-x_{2} \\
-d x_{2}-b x_{3}
\end{array}\right],
\end{aligned}
$$

where

$$
h_{1}=\frac{M_{1}}{M_{1}+M_{2}}, \quad h_{2}=\frac{M_{2}}{M_{1}+M_{2}} .
$$

The configuration of electronic circuit in T-S fuzzy chaotic Lorenz system is shown in Figure 1 and the chaotic behaviors in circuit and MATLAB are shown in Figures 2 and 3. It can be found out that the experimental result in our circuit is actually effective. It means that the nonlinear chaotic systems can be represented by real operations in electronic circuits, not just existing in simulation results.
3.2. Fuzzy Modeling of Chen-Lee System. For Chen-Lee system,

$$
\begin{aligned}
& \dot{y}_{1}=-y_{2} y_{3}+a_{1} y_{1}, \\
& \dot{y}_{2}=y_{1} y_{3}+b_{1} y_{2}, \\
& \dot{y}_{3}=y_{1} y_{2} / 3+c_{1} y_{3},
\end{aligned}
$$

where $a_{1}, b_{1}$, and $c$ are the parameters. When $a_{1}=5$, $b_{1}=-10, c_{1}=-38$, and initial states are $(0.2,0.2,0.2)$, the dynamic behavior is chaotic. Assume that $y_{1} \in\left[-d_{1}, d_{1}\right]$, $y_{2} \in\left[-e_{1}, e_{1}\right]$, and $d_{1}>0, e_{1}>0$, then Chen-Lee system can be exactly represented by T-S fuzzy model as follows.

Rule 1: IF $y_{1}$ is $P_{1}$ and IF $y_{2}$ is $Q_{1}$, THEN

$$
\dot{Y}(t)=B_{1} Y(t) .
$$

Rule 2: IF $y_{1}$ is $P_{1}$ and IF $y_{2}$ is $Q_{2}$, THEN

$$
\dot{Y}(t)=B_{2} Y(t) .
$$

Rule 3: IF $y_{1}$ is $P_{2}$ and IF $y_{2}$ is $Q_{1}$, THEN

$$
\dot{Y}(t)=B_{3} Y(t) .
$$

Rule 4: IF $y_{1}$ is $P_{2}$ and IF $y_{2}$ is $Q_{2}$, THEN

$$
\dot{Y}(t)=B_{4} Y(t),
$$




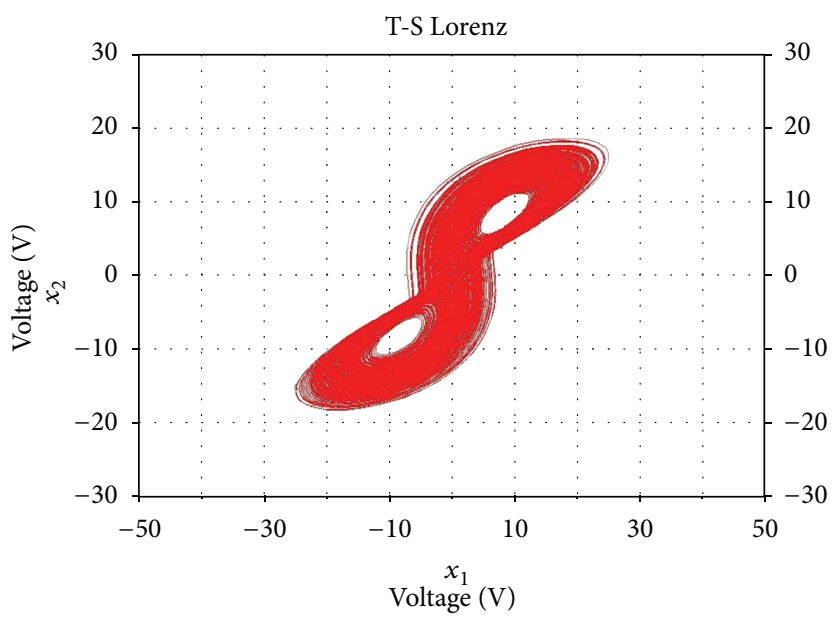

(a)

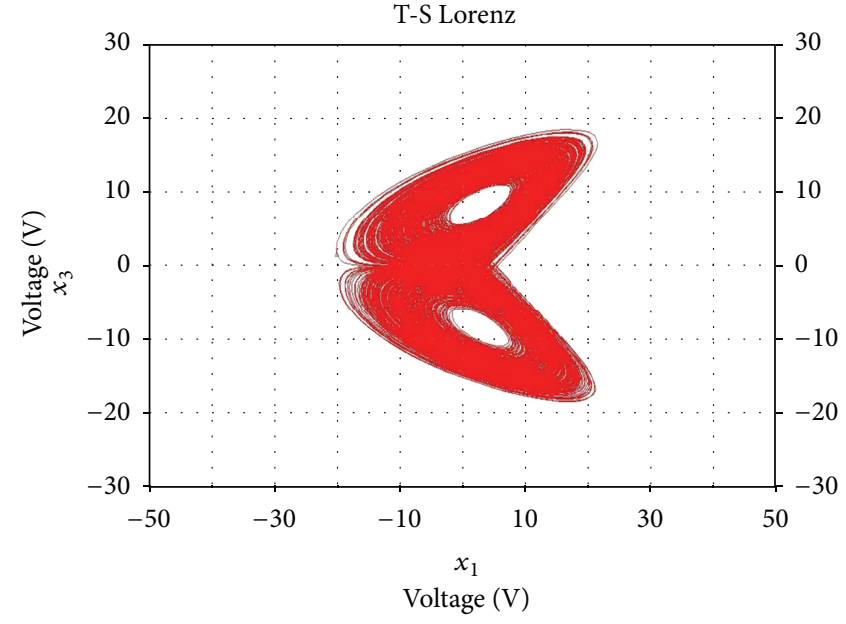

(b)

FIGURE 2: Projection of phase portraits outputs in fuzzy electronic circuit for the Lorenz system.

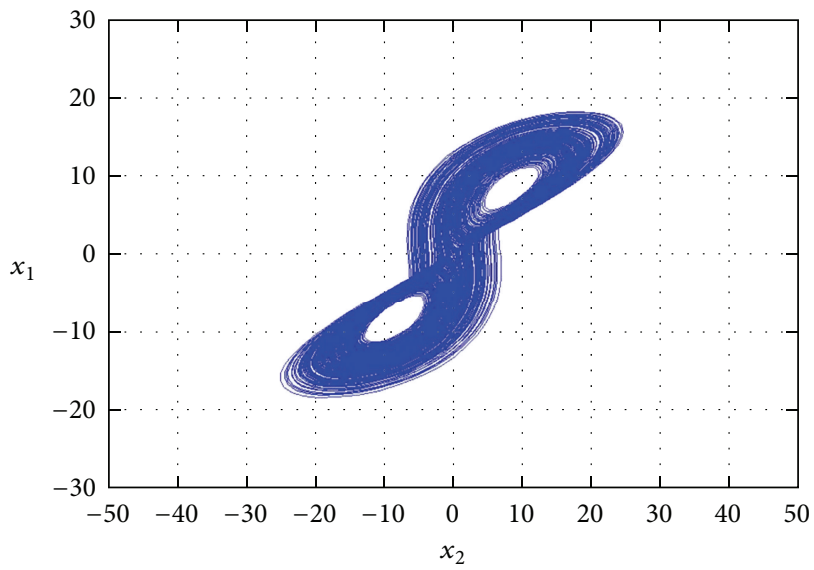

(a)

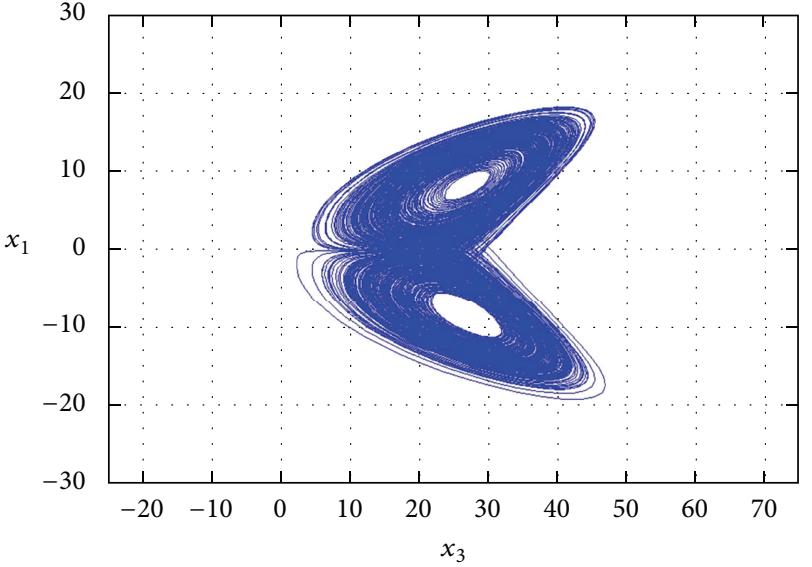

(b)

Figure 3: Projection of phase portraits in MATLAB for fuzzy chaotic Lorenz system.

where

$$
\begin{aligned}
& Y=\left[y_{1}, y_{2}, y_{3}\right]^{T}, \\
& B_{1}=\left[\begin{array}{ccc}
a & 0 & -e \\
0 & b & d \\
0 & \frac{1}{3} d & c
\end{array}\right], \quad B_{2}=\left[\begin{array}{ccc}
a & 0 & e \\
0 & b & d \\
0 & \frac{1}{3} d & c
\end{array}\right] \text {, } \\
& B_{1}=\left[\begin{array}{ccc}
a & 0 & -e \\
0 & b & -d \\
0 & -\frac{1}{3} d & c
\end{array}\right], \quad B_{2}=\left[\begin{array}{ccc}
a & 0 & e \\
0 & b & -d \\
0 & -\frac{1}{3} d & c
\end{array}\right] \text {, } \\
& P_{1}(y)=\frac{1}{2}\left(1+\frac{y_{1}}{d_{1}}\right), \quad P_{2}(y)=\frac{1}{2}\left(1-\frac{y_{1}}{d_{1}}\right) \text {, } \\
& Q_{1}(y)=\frac{1}{2}\left(1+\frac{y_{2}}{e_{1}}\right), \quad Q_{2}(y)=\frac{1}{2}\left(1-\frac{y_{2}}{e_{1}}\right) \text {. }
\end{aligned}
$$

Choose $d_{1}=40$ and $e_{1}=30 . N_{1}$ and $N_{2}$ are fuzzy sets of Chen-Lee system. Here, we call (16) the first linear subsystem under the fuzzy rule and (17) the second linear subsystem under the fuzzy rule. The final output of the fuzzy Chen-Lee system is inferred as follows:

$$
\begin{aligned}
\dot{Z}(t)= & \sum_{i=1}^{2} I_{i} B_{i} Z(t) \\
= & {\left[\begin{array}{c}
I_{1} \\
I_{1} \\
I_{1}
\end{array}\right]^{T}\left[\begin{array}{c}
-e_{1} y_{3}+a_{1} y_{1} \\
d_{1} y_{3}+b_{1} y_{2} \\
d_{1} y_{2} / 3+c_{1} y_{3}
\end{array}\right]+\left[\begin{array}{l}
I_{2} \\
I_{2} \\
I_{2}
\end{array}\right]^{T}\left[\begin{array}{c}
e_{1} y_{3}+a_{1} y_{1} \\
d_{1} y_{3}+b_{1} y_{2} \\
d_{1} y_{2} / 3+c_{1} y_{3}
\end{array}\right] } \\
& +\left[\begin{array}{c}
I_{3} \\
I_{3} \\
I_{3}
\end{array}\right]^{T}\left[\begin{array}{c}
-e_{1} y_{3}+a_{1} y_{1} \\
-d_{1} y_{3}+b_{1} y_{2} \\
-d_{1} y_{2} / 3+c_{1} y_{3}
\end{array}\right] \\
& +\left[\begin{array}{c}
I_{4} \\
I_{4} \\
I_{4}
\end{array}\right]^{T}\left[\begin{array}{c}
e_{1} y_{3}+a_{1} y_{1} \\
-d_{1} y_{3}+b_{1} y_{2} \\
-d_{1} y_{2} / 3+c_{1} y_{3}
\end{array}\right],
\end{aligned}
$$




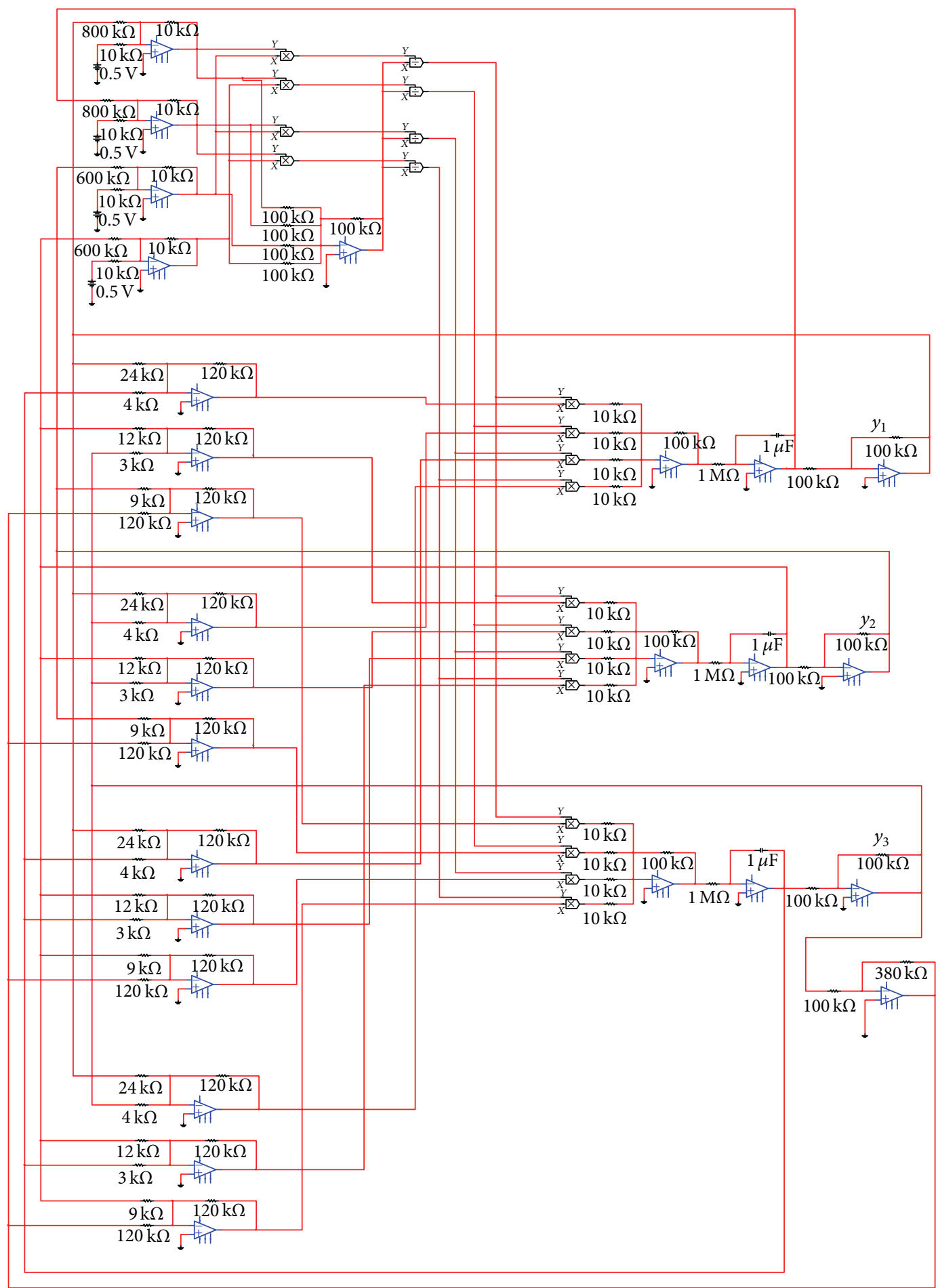

FIgURE 4: The fuzzy electronic circuit for chaotic Chen-Lee system. 


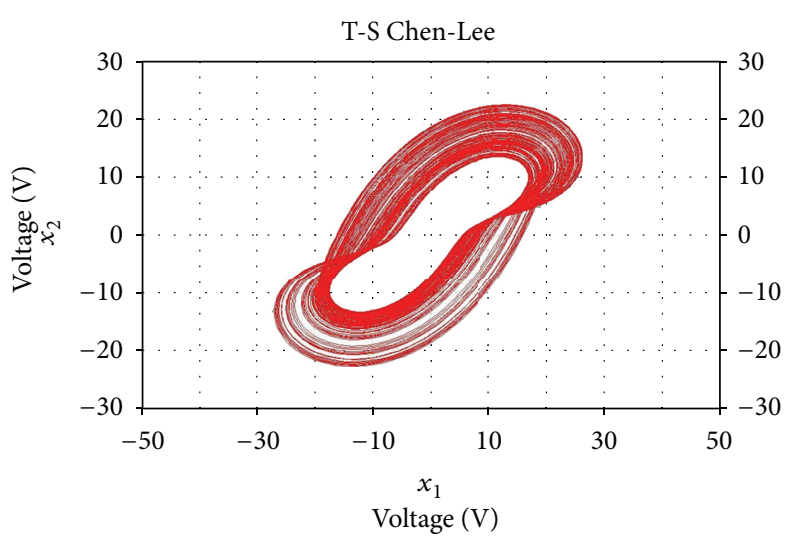

(a)

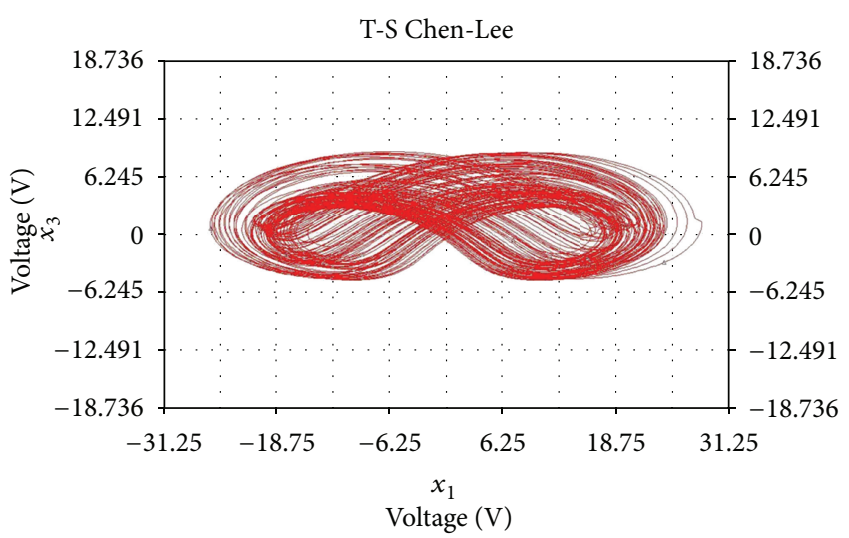

(b)

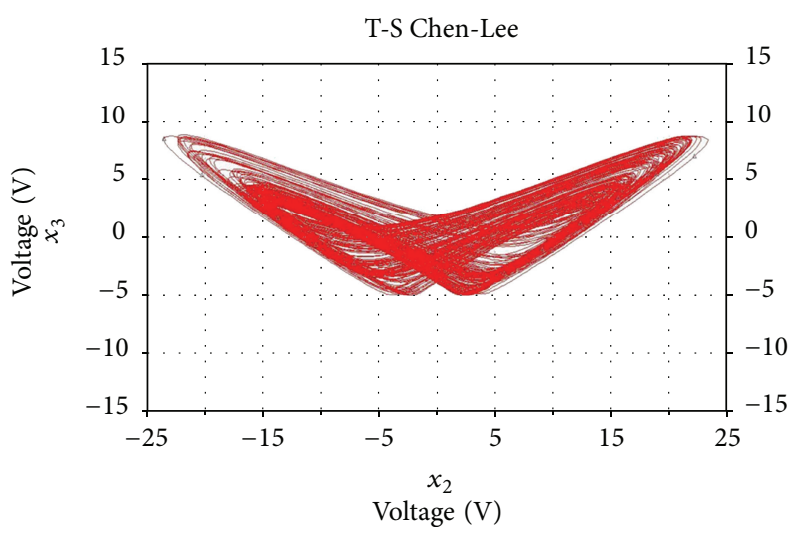

(c)

Figure 5: Projection of phase portraits outputs in fuzzy electronic circuit for Chen-Lee system.

where

$$
\begin{array}{ll}
I_{1}=\frac{P_{1} \times Q_{1}}{P_{1}+P_{2}+Q_{1}+Q_{2}}, & I_{2}=\frac{P_{1} \times Q_{2}}{P_{1}+P_{2}+Q_{1}+Q_{2}}, \\
I_{3}=\frac{P_{2} \times Q_{1}}{P_{1}+P_{2}+Q_{1}+Q_{2}}, & I_{4}=\frac{P_{2} \times Q_{2}}{P_{1}+P_{2}+Q_{1}+Q_{2}} .
\end{array}
$$

The configuration of electronic circuit in T-S fuzzy chaotic Chen-Lee system is shown in Figure 4 and the chaotic behaviors in circuit and MATLAB are shown in Figures 5 and 6. This experimental result in T-S fuzzy chaotic ChenLee system is exactly effective as well.

Two illustrations given in Sections 3.1 and 3.2 all show the agreement between our experimental and MATLAB simulation results. It means the T-S fuzzy model would no longer be just a mathematical tool, it can be applied to electronic circuits for various kinds of applications in practice.

\section{Conclusions}

The implementations of Takagi-Sugeno (T-S) fuzzy chaotic systems on electronic circuits are proposed in this paper. We construct the powerful tool, Takagi-Sugeno (T-S) fuzzy model, on electronic circuit and show good agreement between computer simulations in MATLAB and experimental results in our circuits. Through our effort, the powerful Takagi-Sugeno (T-S) method is more than just a numerical strategy, it can be applied to electronic circuits for various kinds of applications in practice. Implementations of electronic circuits for Takagi-Sugeno (T-S) fuzzy chaotic systems are only the beginning for secure communication and other kinds of applications, this paper also creates both opportunities and challenges. Implementations of novel synchronization or control approaches on electronic circuits in nonlinear research filed would be definitely our future directions to achieve.

\section{Acknowledgments}

This work was supported in part by the UST-UCSD International Center of Excellence in Advanced Bioengineering sponsored by the Taiwan National Science Council I-RiCE Program under Grant no. NSC-101-2911-I-009-101. This work was also by the Aiming for the Top University Plan of National Chiao Tung University, the Ministry of Education, Taiwan, under Contract 101W963, and by the Army Research Laboratory and was accomplished under Cooperative Agreement no. W911NF-10-2-0022. 


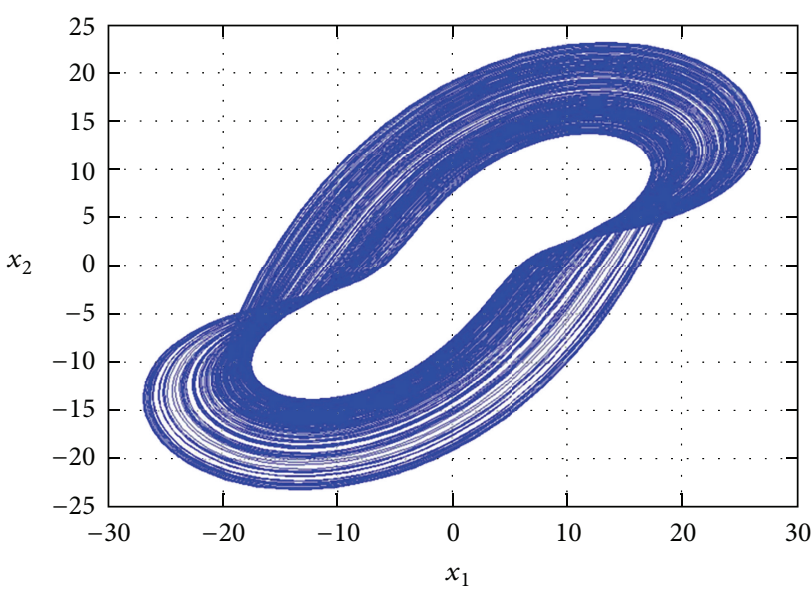

(a)

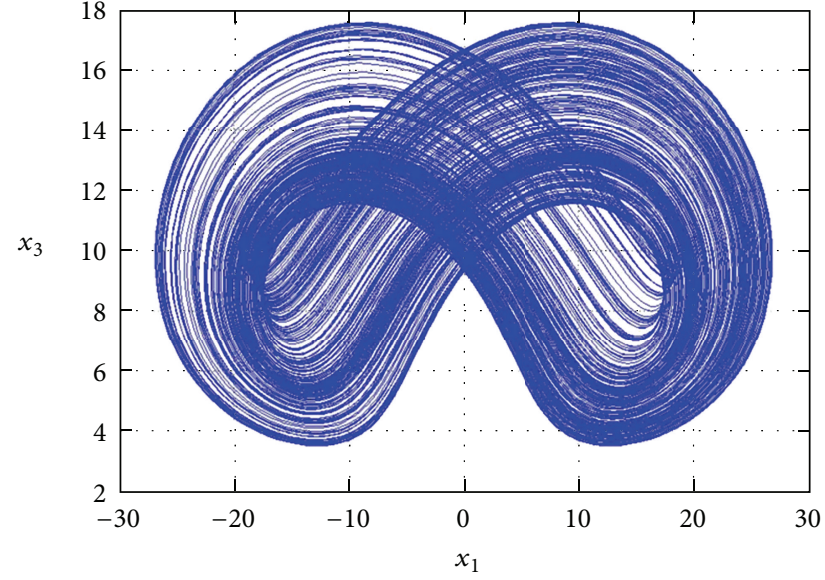

(b)

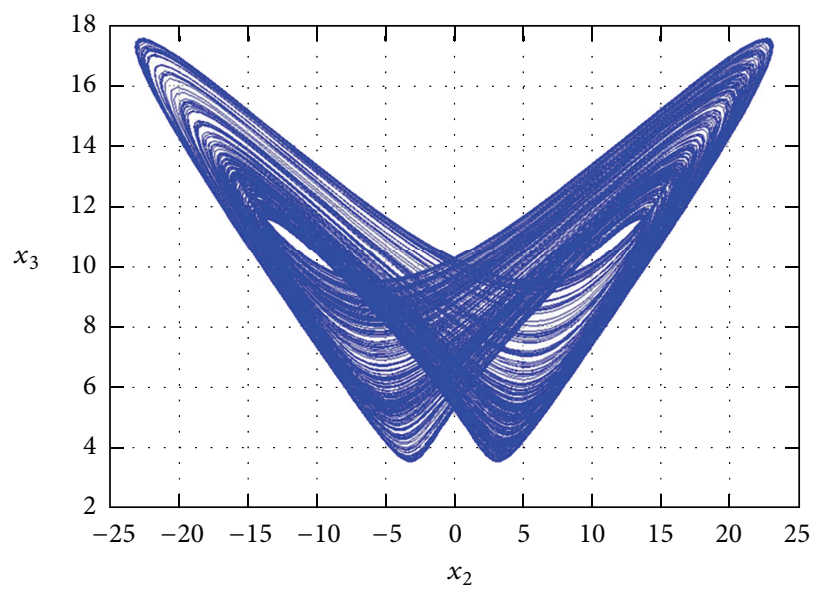

(c)

Figure 6: Projection of phase portraits in MATLAB for fuzzy chaotic Chen-Lee system.

\section{References}

[1] S. Y. Li, C. H. Yang, S. A. Chen, L. W. Ko, and C. T. Lin, "Fuzzy adaptive synchronization of time-reversed chaotic systems via a new adaptive control strategy," Information Sciences, vol. 222, no. 10, pp. 486-500, 2013.

[2] A. Mamandi, M. H. Kargarnovin, and S. Farsi, "Dynamic analysis of a simply supported beam resting on a nonlinear elastic foundation under compressive axial load using nonlinear normal modes techniques under three-to-one internal resonance condition," Nonlinear Dynamics, vol. 70, no. 2, pp. 1147$1172,2012$.

[3] S. Y. Li and Z. M. Ge, "Generating tri-chaos attractors with three positive lyapunov exponents in new four order system via linear coupling," Nonlinear Dynamics, vol. 69, no. 3, pp. 805-816, 2012.

[4] C.-H. Yang, T.-W. Chen, S.-Y. Li, C.-M. Chang, and Z.-M. Ge, "Chaos generalized synchronization of an inertial tachometer with new Mathieu-Van der Pol systems as functional system by GYC partial region stability theory," Communications in Nonlinear Science and Numerical Simulation, vol. 17, no. 3, pp. 1355-1371, 2012.

[5] M. S. H. Chowdhury, I. Hashim, S. Momani, and M. M. Rahman, "Application of multistage homotopy perturbation method to the chaotic Genesio system," Abstract and Applied Analysis, vol. 2012, Article ID 974293, 10 pages, 2012.

[6] Y. Horikawa and H. Kitajima, "Quasiperiodic and exponential transient phase waves and their bifurcations in a ring of unidirectionally coupled parametric oscillators," Nonlinear Dynamics, vol. 70, no. 2, pp. 1079-1094, 2012.

[7] A. Freihat and S. Momani, "Adaptation of differential transform method for the numeric-analytic solution of fractional-order Rössler chaotic and hyperchaotic systems," Abstract and Applied Analysis, vol. 2012, Article ID 934219, 13 pages, 2012.

[8] Z.-M. Ge and S.-Y. Li, "Chaos generalized synchronization of new Mathieu-Van der Pol systems with new Duffing-Van der Pol systems as functional system by GYC partial region stability theory," Applied Mathematical Modelling, vol. 35, no. 11, pp. 5245-5264, 2011.

[9] Z.-M. Ge and S.-Y. Li, "Chaos control of new Mathieu-Van der Pol systems with new Mathieu-Duffing systems as functional system by GYC partial region stability theory," Nonlinear Analysis: Theory, Methods \& Applications, vol. 71, no. 9, pp. 4047-4059, 2009.

[10] C. Yin, S.-M. Zhong, and W.-F. Chen, "Design of sliding mode controller for a class of fractional-order chaotic systems," Communications in Nonlinear Science and Numerical Simulation, vol. 17, no. 1, pp. 356-366, 2012. 
[11] J. Zhao, "Adaptive Q-S synchronization between coupled chaotic systems with stochastic perturbation and delay," Applied Mathematical Modelling, vol. 36, no. 7, pp. 3312-3319, 2012.

[12] M. Villegas, F. Augustin, A. Gilg, A. Hmaidi, and U. Wever, "Application of the polynomial chaos expansion to the simulation of chemical reactors with uncertainties," Mathematics and Computers in Simulation, vol. 82, no. 5, pp. 805-817, 2012.

[13] M. F. Pérez-Polo and M. Pérez-Molina, "Saddle-focus bifurcation and chaotic behavior of a continuous stirred tank reactor using PI control," Chemical Engineering Science, vol. 74, no. 28, pp. 79-92, 2012.

[14] J. Jiao and L. Chen, "The genic mutation on dynamics of a predator-prey system with impulsive effect," Nonlinear Dynamics, vol. 70, no. 1, pp. 141-153, 2012.

[15] T. Wang, N. Jia, and K. Wang, "A novel GCM chaotic neural network for information processing," Communications in Nonlinear Science and Numerical Simulation, vol. 17, no. 2, pp. 48464855, 2012.

[16] N. X. Quyen, V. V. Yem, and T. M. Hoang, "A chaotic pulse-time modulation method for digital communication," Abstract and Applied Analysis, vol. 2012, Article ID 835304, 15 pages, 2012.

[17] T. Wang, N. Jia, and K. Wang, "A novel GCM chaotic neural network for information processing," Communications in Nonlinear Science and Numerical Simulation, vol. 17, no. 12, pp. 4846-4855, 2012.

[18] S. Wang and H. Yao, "The effect of control strength on lag synchronization of nonlinear coupled complex networks," Abstract and Applied Analysis, vol. 2012, Article ID 810364, 11 pages, 2012.

[19] J. L. Mata-Machuca, R. Martínez-Guerra, R. Aguilar-López, and C. Aguilar-Ibañez, "A chaotic system in synchronization and secure communications," Communications in Nonlinear Science and Numerical Simulation, vol. 17, no. 4, pp. 1706-1713, 2012.

[20] Y.-Y. Hou, H.-C. Chen, J.-F. Chang, J.-J. Yan, and T.-L. Liao, "Design and implementation of the Sprott chaotic secure digital communication systems," Applied Mathematics and Computation, vol. 218, no. 24, pp. 11799-11805, 2012.

[21] E. N. Lorenz, "Deterministic non-periodic flows," Journal of the Atmospheric Science, vol. 20, no. 2, pp. 130-141, 1963.

[22] S. Li, Y. Li, B. Liu, and T. Murray, "Model-free control of Lorenz chaos using an approximate optimal control strategy," Communications in Nonlinear Science and Numerical Simulation, vol. 17, no. 12, pp. 4891-4900, 2012.

[23] A. M. A. El-Sayed, E. Ahmed, and H. A. A. El-Saka, "Dynamic properties of the fractional-order logistic equation of complex variables," Abstract and Applied Analysis, vol. 2012, Article ID 251715, 12 pages, 2012.

[24] H.-K. Chen, "Synchronization of two different chaotic systems: a new system and each of the dynamical systems Lorenz, Chen and Lü," Chaos, Solitons \& Fractals, vol. 25, no. 5, pp. 1049-1056, 2005.

[25] Q. Bi and Z. Zhang, "Bursting phenomena as well as the bifurcation mechanism in controlled Lorenz oscillator with two time scales," Physics Letters A, vol. 375, no. 8, pp. 1183-1190, 2011.

[26] F. Oumlzkaynak and A. B. Oumlzer, "A method for designing strong S-Boxes based on chaotic Lorenz system," Physics Letters A, vol. 374, no. 36, pp. 3733-3738, 2010.

[27] S. Y. Li and Z. M. Ge, "Generalized synchronization of chaotic systems with different orders by fuzzy logic constant controller," Expert Systems With Applications, vol. 37, no. 3, pp. 1357-1370, 2011.
[28] H.-K. Chen and C.-I. Lee, "Anti-control of chaos in rigid body motion," Chaos, Solitons and Fractals, vol. 21, no. 4, pp. 957-965, 2004.

[29] L. M. Tam and W. M. SiTou, "Parametric study of the fractional order Chen-Lee System," Chaos, Solitons \& Fractals, vol. 37, no. 3, pp. 817-826, 2008.

[30] L. M. Tam, J. H. Chen, and H. K. Chen, "Generation of hyperchaos from the Chen-Lee system via sinusoidal perturbation," Chaos, Solitons \& Fractals, vol. 38, no. 3, pp. 826-839, 2008.

[31] J. H. Chen, "Controlling chaos and chaotification in the ChenLee system by multiple time delays," Chaos, Solitons \& Fractals, vol. 36, no. 4, pp. 843-852, 2008.

[32] L. A. Zadeh, "Fuzzy sets," Information and Computation, vol. 8, no. 3, pp. 338-353, 1965.

[33] L. A. Zadeh, "Fuzzy logic," IEEE Computer, vol. 21, no. 4, pp. 83-93, 1988.

[34] T. Takagi and M. Sugeno, "Fuzzy identification of systems and its applications to modeling and control," IEEE Transactions on Systems, Man and Cybernetics, vol. 15, no. 1, pp. 116-132, 1985.

[35] N. Zimic and M. Mraz, "Decomposition of a complex fuzzy controller for the truck-and-trailer reverse parking problem," Mathematical and Computer Modelling, vol. 43, no. 5-6, pp. 632645, 2006.

[36] Y.-W. Wang, Z.-H. Guan, and H. O. Wang, "LMI-based fuzzy stability and synchronization of Chen's system," Physics Letters A, vol. 320, no. 2-3, pp. 154-159, 2003.

[37] S. Y. Li, "Chaos control of new Mathieu-van der Pol systems by fuzzy logic constant controllers," Applied Soft Computing, vol. 11, no. 8, pp. 4474-4487, 2011.

[38] Z. M. Ge and S. Y. Li, "Fuzzy modeling and synchronization of chaotic Quantum cellular neural networks nano system via a novel fuzzy model and its implementation on electronic circuits," Journal of Computational and Theoretical Nanoscience, vol. 7, no. 11, pp. 2453-2462, 2010.

[39] C.-J. Lin and Y.-J. Xu, "A hybrid evolutionary learning algorithm for TSK-type fuzzy model design," Mathematical and Computer Modelling, vol. 43, no. 5-6, pp. 563-581, 2006.

[40] S. S. Jin and Y.-H. Lee, "Fuzzy stability of a functional equation deriving from quadratic and additive mappings," Abstract and Applied Analysis, vol. 2011, Article ID 534120, 15 pages, 2011.

[41] S. Y. Li and Z. M. Ge, "Fuzzy modeling and synchronization of two totally different chaotic systems via novel fuzzy model," IEEE Transactions on Systems, Man, and Cybernetics B, vol. 41, no. 4, pp. 1015-1026, 2011.

[42] M. Şengönül and Z. Zararsız, "Some additions to the fuzzy convergent and fuzzy bounded sequence spaces of fuzzy numbers," Abstract and Applied Analysis, vol. 2011, Article ID 837584, 12 pages, 2011.

[43] N. H. Tran, A. Ravoof, T. Nguyen, and K. Tran, "Modelling of type I fracture network: objective function formulation by fuzzy sensitivity analysis," Mathematical and Computer Modelling, vol. 49, no. 7-8, pp. 1283-1287, 2009. 


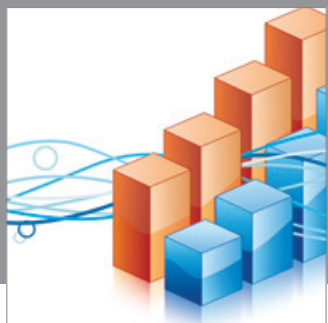

Advances in

Operations Research

mansans

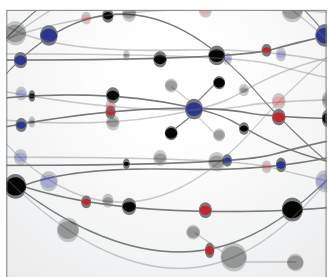

The Scientific World Journal
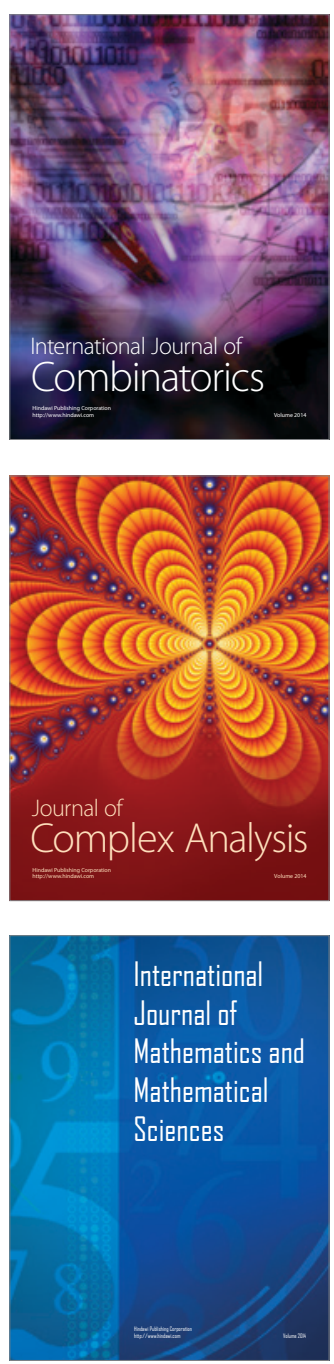
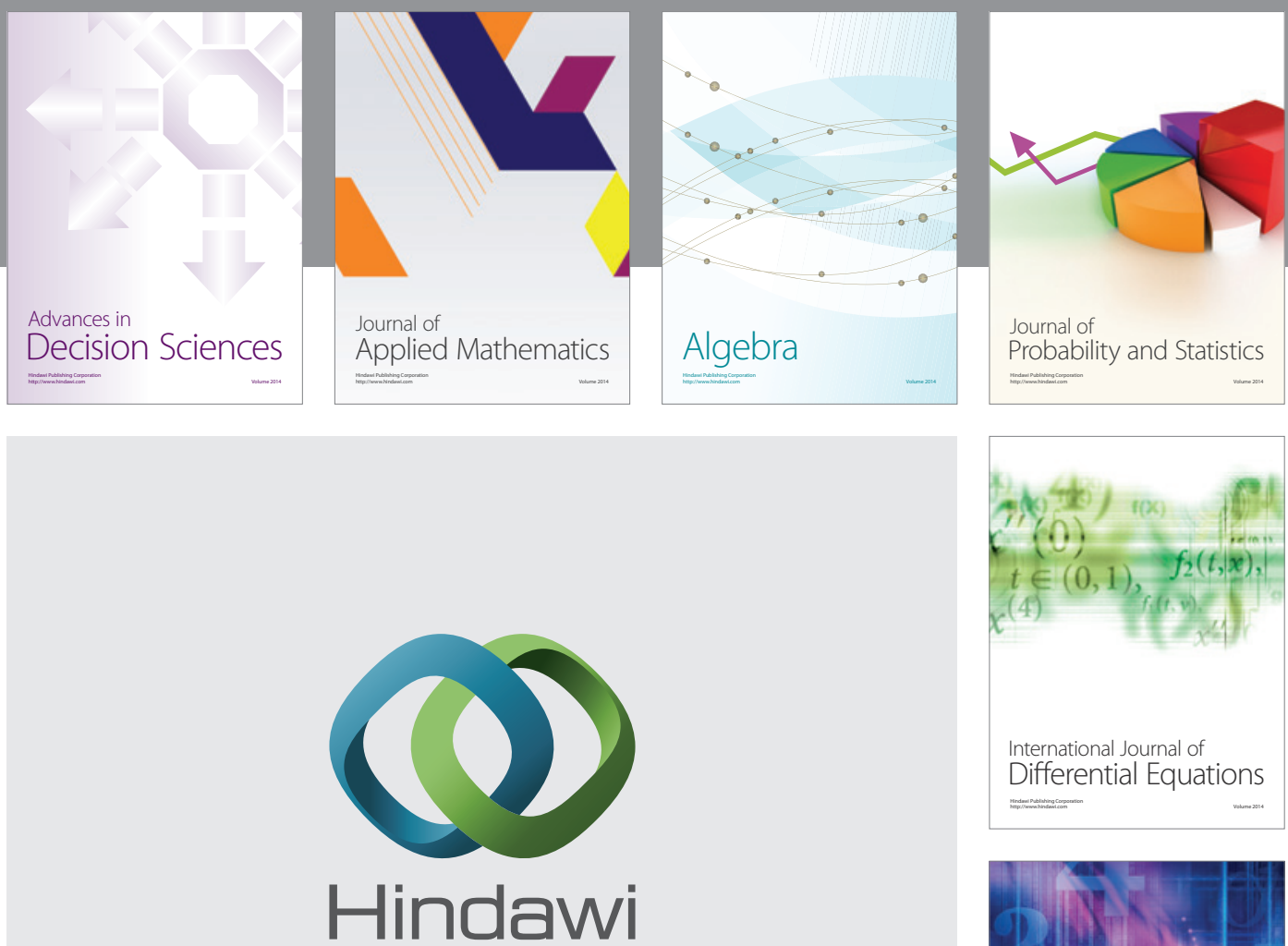

Submit your manuscripts at http://www.hindawi.com
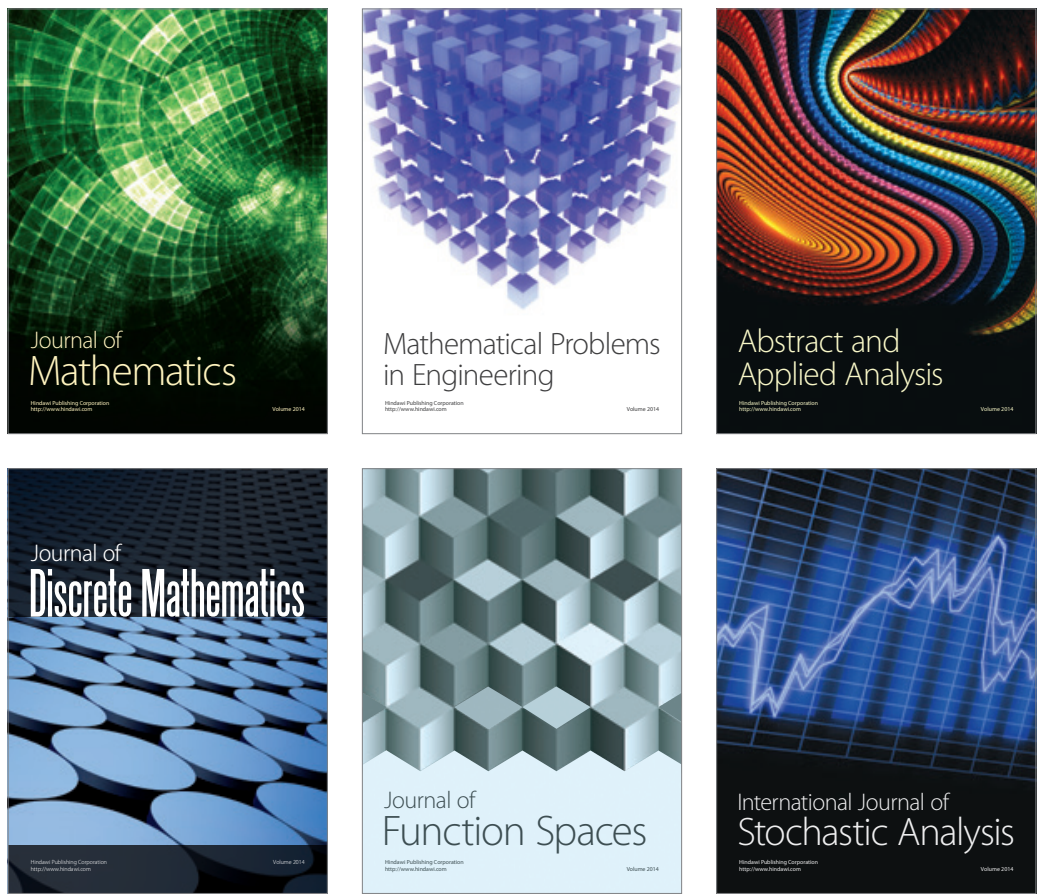

Journal of

Function Spaces

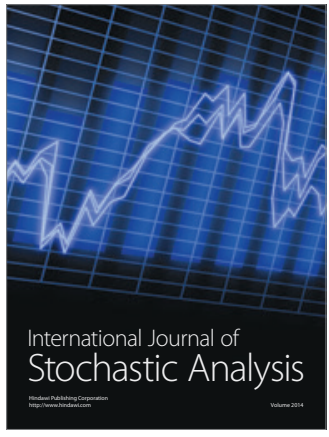

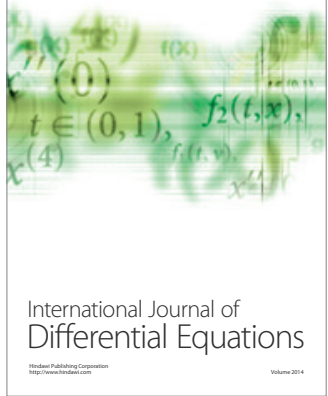
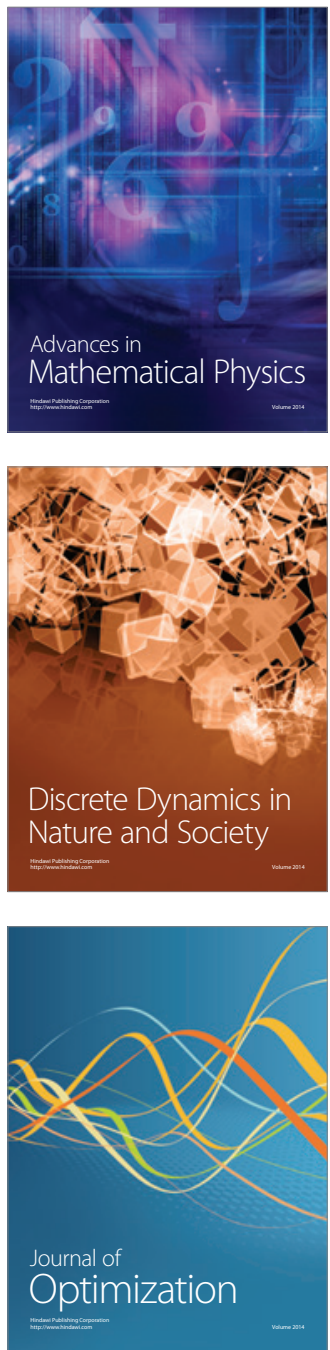